\title{
Finite deformations of elastic fibre-reinforced materials
}

\section{Michael Stuart Craig}

of the problems dealing with finite deformations of elastic fibrereinforced materials, two types are considered in this thesis. One is the boundary value problem for incompressible materials and the other is the problem of finding controllable deformations for compressible materials which are isotropic but for the presence of the fibres. Controllable deformations are ones which may be maintained in equilibrium under the action of boundary tractions only in any material of the given class.

In considering boundary value problems for finite, plane deformations of incompressible fibre-reinforced materials, it is important to note that the governing equations are hyperbolic instead of elliptic for the nonreinforced case. The types of boundary data one would like to impose on the reinforced composites are the same as those imposed on the nonreinforced materials. Hence, for these types of boundary conditions, there is no guarantee that the normal displacement, stress, and mixed boundary value problems are well posed for fibre-reinforced materials.

Because of the kinematic constraints introduced by the fibres and the incompressibility condition, displacement boundary data, and mixed boundary data may not be arbitrarily prescribed on a closed boundary. It is found that the segments of a boundary on which data may be prescribed depend on the actual data given. Once consistent displacement and stress data have been prescribed, consistent deformations are found to exist and these may be actually calculated in some cases. All the above is valid whatever the constitutive relation of the material.

Received 17 August 1977. Thesis submitted to the University of Queensland, February 1977. Degree approved, August 1977. Supervisor: Dr V.G. Hart. 
The pure stress boundary value problem on the other hand appears to be well-posed. For a certain Mooney-type material, reinforced with straight, parallel fibres initially, this problem may be converted to a consistent displacement boundary value problem (which may be solved explicitly) by the solution of a highly non-linear set of equations. The solution of these non-linear equations is found for the trivial case of zero stress and a perturbation scheme is set up for the case where the given stress is "small". By this means some exact and approximate stress boundary value problems are solved.

The other problem we consider is the finding of three-dimensional controllable deformations. Here we show that, if the fibres are to be straight in the final configuration, then a deformation must lie in one of several categories if it is to be controllable. In the case where the fibres are not straight in the final configuration, two controllable deformations are found, but the question remains open if any more exist. 